\title{
Development of hybrid model based on Lattice Boltzmann Method and Cellular Automata devoted for phase transformation - simulation of heat flow with consideration of enthalpy
}

\author{
Eukasz Lach $^{1, *}$, Robert Straka ${ }^{1}$, and Dmytro Svyetlichnyy ${ }^{1}$ \\ ${ }^{1}$ AGH University of Science and Technology, Faculty of Metals Engineering and Industrial Computer Science, al. Mickiewicza 30, \\ Kraków, Poland
}

\begin{abstract}
In heat treatment of materials, the phase transformation is an important phenomenon, which determines the final microstructure. The microstructure of different materials described by such parameters as morphology, grain size, phase fraction and their spatial distribution, largely effects on the mechanical and functional properties of final products. The subject of the work is a development of a hybrid model based on CA and Lattice Boltzmann method (LBM) for modeling of the diffusion phase transformation. The model has a modular structure and simulates three basic phenomena: diffusion, heat flow and phase transformation. The objective of the paper is a presentation of module of the hybrid model for simulation of heat flow with considering of enthalpy of transformation. This is one of the stages in the development of the model and obtained results will be used in a combined solution of heat transfer and diffusion during the modeling of diffusion phase transformations. Lately, the model will be extended to three dimensions and will use hybrid computational systems (CPU and GPU). CA and LBM are used in the model as follows. LBM is used for modeling of heat flow, while CA is used for modeling of microstructure evolution during the phase transformation. The main factors considered in the model are the enthalpy of transformation and heat transfer. The paper presents the results of the modeling of the new phase growth determined by different values of overcooling affecting on different values in the enthalpy of transformation. The heat flow is simulated and the results for some modeling variants are shown. Examples of simulation results obtained from the modeling are presented in the form of images, which present the growth of new phase and temperature distributions.
\end{abstract}

\section{Introduction}

A A fundamental principle of materials engineering is that the microstructure affects on the physical properties and behaviour of a material. Microstructure is formed through a variety of different processes and in the most cases it is created during the phase transformation under effects of the temperature and/or pressure. Microstructures can be created through deformation or processing of the material (e.g. rolling, welding) and also artificially by combining different materials to form a composite material. Because the microstructure can be described as a composition of phases presented in a material (their morphology, distribution and internal structure), the phenomenon of the phase transformation plays an important role in the formation of the microstructure. Many of important material properties can be engineered by control of its solid state phase transformations, so experimental and modeling studies related to this type of the transformations are intensively realized. Thus, a modeling of the phase transformations is a primordial task for development of new materials with new properties, for improving the performance of existing materials, or designing new processes. Recently, many analytical and numerical methods are used for modeling of phase transformation, and various aspects of transformation are considered, for example nucleation of grains of new phase $[1,2]$, grain growth $[3,4]$, diffusion [5-7], etc. New numerical models of phase transformations are created based on the cellular automata (CA), finite difference method (FDM), phase field $(\mathrm{PF})$ and others methods. Two-dimensional cellular automata model was used by An et al. [8] to simulate the ferrite-austenite transformation in binary low-carbon steels, while the Varma et al. [9] used two-dimensional diffusion based model to describe transformation of austenite into ferrite and pearlite under continuous cooling conditions. Three dimensional CA model was developed for example by Svyetlichnyy et al. [10] and austenite into ferrite and perlite transformation was modeled. FDM method was used for example by Wang et al. [11] for the verification of two models of phase transitions described by various mechanisms of the new phase growth and by Singh [12] for modeling the allotropic transformation of titanium as a result of high temperature oxidation. PF method was used for study the

\footnotetext{
Corresponding author: lach@agh.edu.pl
} 
cubic to monoclinic martensitic phase transformation in nickel-titanium (NiTi) shape memory alloys [13] and for modeling of martensitic transformation [14].

Taking into account the modeling of the diffusion phase transformations and existing models, it can be seen that a lot of limitations exists for example in relation to the choice of the shape of growing grains and the introduction of variable grain growth rate, and only some models allow for accurately modeling of diffusion phenomena and there are a few comprehensive studies, which combine the final microstructure with the actual conditions of its formation. Therefore, the objective of the work is a development of a new hybrid model based on Lattice Boltzmann Method and Cellular Automata for modeling of the diffusion phase transformations. The model will provide completely new opportunities and will allow to comprehensive modeling of the diffusion phase transformations in many processes, which consist of a heating, annealing and cooling.

The paper presents the structure of the hybrid model and its basic modules - the main attention is paid on the temperature and microstructure evolution modules, and the simulation of heat flow with consideration of the enthalpy of transformation. It is one of the stages in development of the model. Examples of the simulation results of the heat flow modeling using the LBM and CA are also presented.

\section{Hybrid LBM and CA model - heat flow module}

The part of the structure of the hybrid model of the phase transformation that relates to heat flow is shown in Fig. 1. The whole model is based on two modeling methods. The first method is the LBM method and the second one is cellular automata. In the main part, the model is based on the LBM method, which is a relatively new method and it is just beginning to be widely used in modeling. Taking into account that this method has got excellent numerical stability and constitutive versatility, it can play an essential role as a simulation tool for modeling of different phenomena and processes. Recently, LBM has been used for different issues, for example: modeling and simulation of liquid jet breakup [15], modeling dendritic solidification under forced and natural convection [16], modeling of thermal conduction in composites with thermal contact resistance [17], modeling of transport phenomena in fuel cells and flow batteries [18], modeling of the additive layer manufacturing [19]. Lattice Boltzmann method consider flows to be composed of a collection of pseudo-particles that are represented by a velocity distribution function, so it can be applied for modeling of different variants of flows, as well as phase transformations in flows. Taking into account the advantages of the LBM method and its applicability in the flow modeling, the heat flow model with consideration of enthalpy is based on this method. One of the most effective methods for modeling of microstructure evolution during various processes is cellular automata. Models based on cellular automata allow to take into account several simultaneously occurring phenomena, bringing modeling closer to real processes. CA allow for modeling of dynamic processes occurring in the materials, for example solidification [20], static [21] and dynamic recrystallization [22], phase transformation [10]. CA can be also used for modeling of phenomena in complex processes, such as additive layer manufacturing of orthopaedic implants [23], flat rolling [24] and shape rolling [25]. Taking into account the advantages of cellular automata, this method is used in the microstructure evolution model for modeling of nucleation and grain boundary movement. Since the basic structure of the LBM method is the same as in the synchronous automata (LBM originates from the variant of CA application known as Lattice Gas Automata), it is also an ideal platform for realizing combinations of these two methods in one modeling solution. The model can be also considered as a combined solution for heat flow, diffusion, and grain growth. The main modules considered in the model and also their interactions are shown in Fig.2. Taking into account this scheme, the main module considered in the paper is the temperature module and its connection with the microstructure module, marked by dashed rectangle. As can be seen on the scheme, the temperature influences directly on the nucleation of new grains and grain boundary movements, while the enthalpy of transformation is associated with the temperature. The next section presents the results of modeling for the analyzed issues. The diffusion module and its connection with the microstructure module have been developed at the previous stage of work (unpublished manuscript).

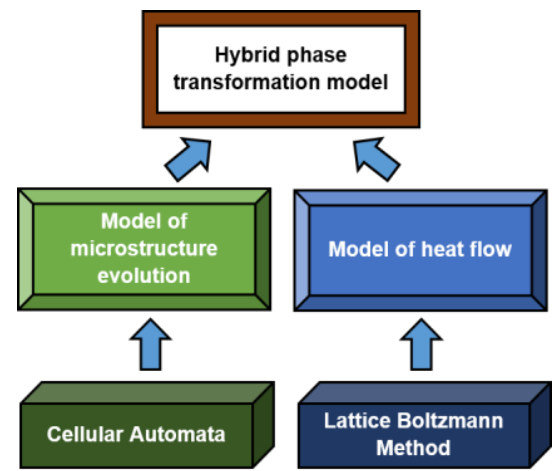

Fig. 1. Structure of hybrid model in part related to heat flow.

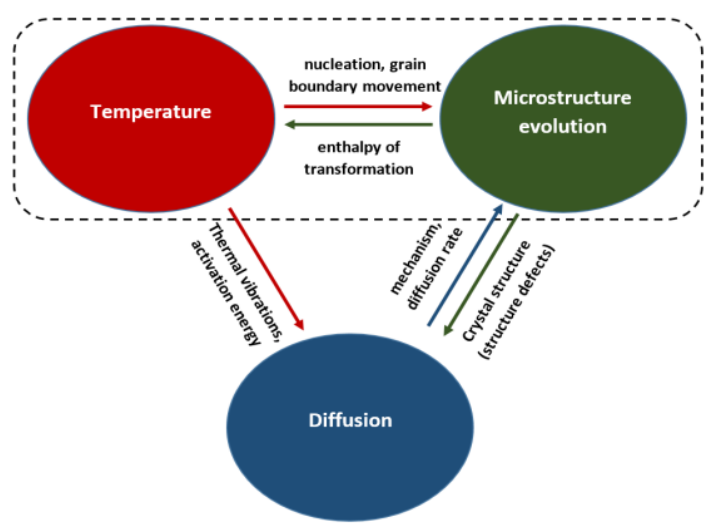

Fig. 2. The main modules considered in the model. 


\section{Analysis and modeling}

On this stage of model development, several variants of heat flow with consideration of enthalpy were modeled using LBM algorithm. The results obtained from the heat flow model were directly linked with the microstructure evolution model, based on cellular automata. Because of there are no applications based on CA and LBM for solution the problem mentioned above, the one dimensional model was developed at first to obtain the preliminary results and verify the correctness of the proposed approach. The 1D Fourier equation describing the heat flow was used in the calculations:

$$
\frac{1}{\alpha} \frac{\partial T}{\partial t}=\frac{\partial^{2} T}{\partial x^{2}}
$$

where: $T$ - temperature, $\alpha$ - thermal diffusivity.

The lattice Boltzmann - BGK (Bhatnagar-GrossKrook) model with single-relaxation-time was used for modeling. Then, evolution of distribution function can be described by the following expression:

$$
f_{k}(x+\Delta x, t+\Delta t)-f_{k}(x, t)=-\frac{\Delta t}{\tau}\left[f_{k}(x, t)-f_{k}^{e q}(x, t)\right]
$$

where: $f_{k}(x, t)$ and $f_{k}^{e q}(x, t)$ are the particle and equilibrium distribution functions at $(x, t)$ and $\tau$ is the single-relaxation-time parameter that controls the rate of solution approach to local equilibrium $f_{k}^{e q}$.

The equilibrium distribution function $f_{k}^{e q}$ :

$$
f_{k}^{e q}=w_{k} \phi(x, t)
$$

where $w_{k}$ stands for the weighting factor in the direction $k$ and $\phi$ is the dependent variable (temperature $T$ in the presented task).

According to the equation (2) two steps can be considered:

collision:

$$
f_{k}(x, t+\Delta t)=f_{k}(x, t)[1-\omega]+\omega f_{k}^{e q}(x, t)
$$

where: $\omega=\Delta t / \tau$.

streaming:

$$
f_{k}(x+\Delta x, t+\Delta t)=f_{k}(x, t+\Delta t)
$$

Fig. 3 shows D1Q2 scheme, which was applied for modeling. The lattice velocities $e_{i}$ and corresponding lattice factors for D1Q2 scheme are given as: $e_{1}=1, e_{2}=$ $-1, w_{1}=1 / 2, w_{2}=1 / 2$.

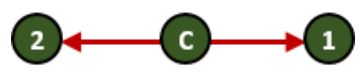

Fig. 3. D1Q2 LBM lattice.

The initial states for nodes in the calculation grid and their update during the transformation are presented in Fig. 4. Taking into account the type of state, the node can be in one of three states: Austenite (A), Interface (I) and Ferrite (F). Dark red nodes represents the austenite, yellow - interface and gray - ferrite.

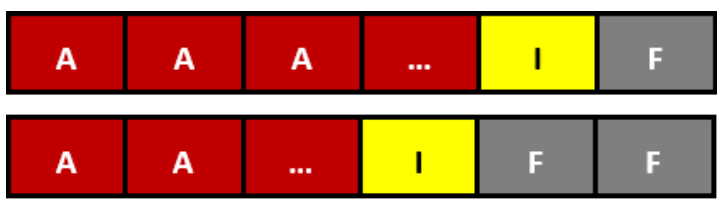

Fig. 4. The changes of states during the transformation.

The calculation algorithm used in the heat flow model includes the following repeated steps:

1. Calculation of a boundary velocity $v: v=k_{v}\left(T_{p}-T_{i}\right)$, where: $k_{v}$ - velocity coefficient, $T_{p}$ - temperature of phase transformation, $T_{i}$ - interface temperature, 2. Determination of the quantity, mass or volume of the transformed material: $\Delta \varphi=v \Delta t$.

3. Determination of a fraction of new phase in interface node: $\varphi_{t}=\varphi_{t-1}+\Delta \varphi_{i}$.

4. Fraction checking:

4a) if $\varphi_{t}<1 \Rightarrow$ go to point 5 ,

4b) if $\varphi_{t} \geq 1$ then:

$-\varphi_{t}^{\prime}=1$,

- change the state of node from I to $F$ and the neighboring node from $\mathrm{A}$ to I,

- set the value of the fraction for the new I:

$\varphi=\varphi_{t}-\varphi_{t}{ }^{\prime}, \Delta \varphi_{i+1}=\varphi_{t}-1$,

- the $\Delta \varphi$ for the old I node:

$\Delta \varphi_{N}=1-\varphi_{t-1}=\Delta \varphi_{i}-\Delta \varphi_{i+1}$

5. Calculation of heat source during the transformation: $Q=k_{q} \Delta \varphi$, where: $k_{q}-$ specific enthalpy, 6. Calculation of new temperature: $T=\Sigma f_{i}+Q$,

7. Determination of the equilibrium distribution functions $f^{q q}(3)$,

8. Collision - determination fout (Eq.4), 9. Streaming - transfer of the distribution function to the neighboring nodes, (Eq.5)

10. Consideration of boundary conditions.

\section{Results and discussion}

The parameters used for modeling in the first case were as follows: number of nodes - nodes $=100$, number of time steps - steps $=300, \Delta x=1, \Delta t=1, k_{v}=0.006$, $k_{q}=25$. For the calculations the following relationship

between the relaxation parameter $(\omega)$ and thermal

diffusivity $(\alpha)$ was used:

$$
\omega=1 /\left(\frac{\alpha}{\left(\Delta t *\left(\frac{\Delta x}{\Delta t}\right)^{2}\right)}+0.5\right) .
$$

Bounce back boundary conditions were applied for the edges. The initial temperature for all nodes was set to $750^{\circ} \mathrm{C}$, while the temperature of phase transformation was equal to $800^{\circ} \mathrm{C}$. The initial states for the nodes were the same as in Fig. 4. Fig. 5 shows three different variants of interface movement and growth of new phase depending on the value of thermal diffusivity $\alpha$ (time is on $y$ axis). The thermal diffusivity has an influence on the temperature distribution and for higher value of $\alpha$, the heat transfer is faster and affects on the rate of phase transformation as can be seen in the presented images. 


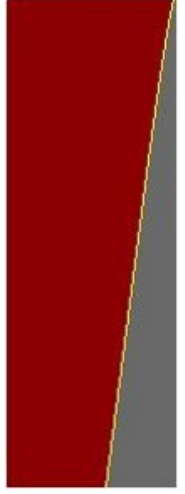

(a)

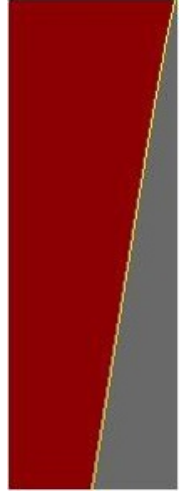

(b)

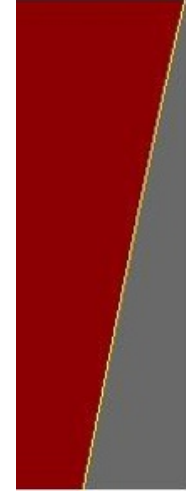

(c)
Fig. 5. Growth of new phase depending on the thermal diffusivity $\alpha$ : a) 0.25 , b) 0.5 , c) 1 .

The next presented results were obtained with the same base parameters as in the first case, and the effect of change of $k_{q}$ coefficient on temperature and rate of phase transformation was studied. The calculations were realized with the same $\alpha=0.5$. Fig. 6 shows the growth of new phase for three different values of $k_{q}$. For lower values of $k_{q}$, the rate of phase tansformation is higher, which is related with the fact that the temperature in the interface grows slower and this has an impact on higher values of boundary velocity. The temperature distributions after 200 time steps are presented in Fig. 7. As can be seen, a higher value of $k_{q}$ gives a higher temperature.

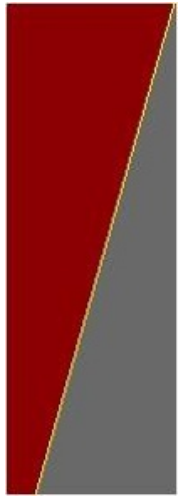

(a)

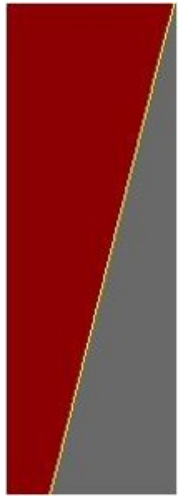

(b)

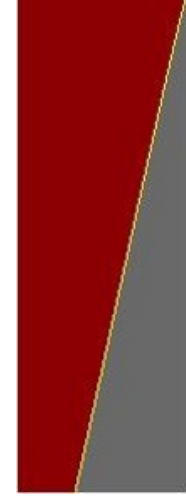

(c)
Fig. 6. Growth of new phase depending on $k_{q}$ : a) 5 , b) 10 , c) 15 .

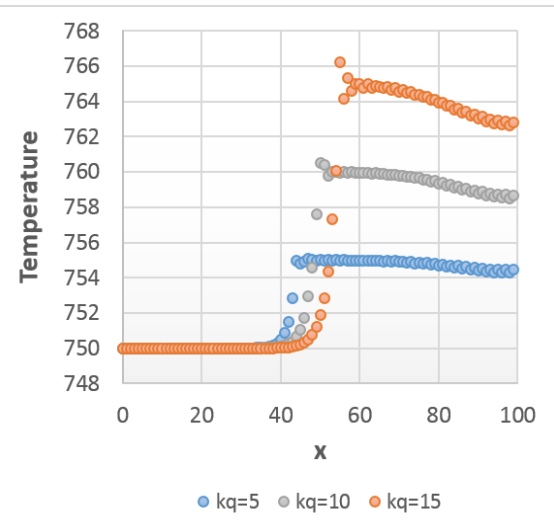

Fig. 7. Temperature distributions after 200 time steps for different value of $k_{q}:$ a) 5, b) 10 , c) 15 .
The influence of coefficient $k_{v}$ was modeled in the last variant. The base simulation parameters were the same as in the first variant, $\alpha=0.5$ and $k_{q}=25$. The simulation results for different value of $k_{v}$ are presented in Fig. 8. Because $k_{v}$ is used for the calculation of velocity, which determines the quantity of the transformed matter, this coefficient influences on the rate of the transformation.

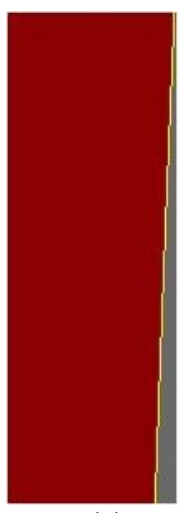

(a)

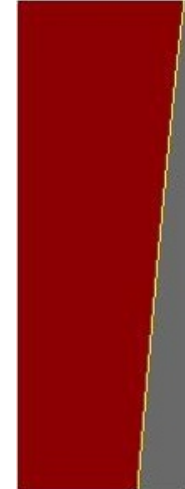

(b)

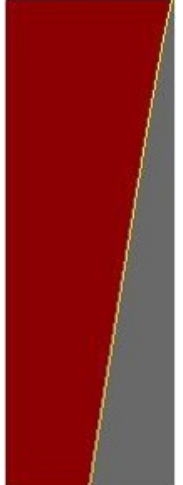

(c)
Fig. 8. Growth of new phase depending on $k_{v}$ : a) 0.001 , b) 0.003, c) 0.006 .

Taking into account the presented results it can be seen that the new phase grows due to the movement of phase interface, while the $\alpha, k_{v}$ and $k_{q}$ coefficients effect on the velocity of interface movement. Determination of the proper and optimal value of $k_{q}$ and $k_{v}$ will be important during the modeling in relation to the real conditions. The appropriate values of these coefficients will be determined based on the results of experimental studies.

\section{Conclusions}

A new hybrid model of diffusion phase transformation in the part related to heat flow was presented in the paper. A connection was established between the two main modules considered in the model: temperature and microstructure evolution module. The use of Lattice Boltzmann Method and Cellular Automata in the model creates completely new possibilities for modeling of phase transformations and in comparison with commonly used approaches significantly simplifies interface and interaction between different parts of the model. The modeling scheme and calculation algorithm were presented and examples simulation results of heat flow with consideration of enthalpy of transformation were shown. The hybrid model in the part related to diffusion has been developed at the previous stage of work (manuscript is submitted to a journal), currently allowing for 1D simulations and now it is possible to combine it with the developed and presented model of heat flow. 2D model of heat flow is also being developed at the current stage of model development and modeling results will be presented in the extended version of the paper. At the final stage of research, the model will be 3D and will use hybrid computational systems (CPU and GPU). 
Acknowledgments: Support of the Polish National Science Centre is greatly appreciated (Grant No. 2016/21/D/ST8/01690).

\section{References}

[1] T.W. Heo, L.-Q. Chen, JOM. 66, 1520 (2014)

[2] D. Zhang, Y. Yin, J. Zhou, Z. Tu, Arch. Foundry Eng. 17, 155 (2017)

[3] L. Feng, B. Jia, C. Zhu, G. An, R. Xiao, X. Feng, Chinese Phys. B. 26, 80504 (2017)

[4] S.E. Offerman, N.H. van Dijk, J. Sietsma, S. Grigull, E.M. Lauridsen, L. Margulies, H.F. Poulsen, M.T. Rekveldt, S. van der Zwaag, Science. 298, 1003 (2002)

[5] M. Chen, H. Alimadadi, S. Molin, L. Zhang, N. Ta, P. V. Hendriksen, R. Kiebach, Y. Du, J. Electrochem. Soc. 164, F1005 (2017)

[6] E. Gamsjäger, F.D. Fischer, J. Svoboda, J. Eng. Mater. Technol. 125, 22 (2003)

[7] K. Nakajima, M. Apel, I. Steinbach, Acta Mater. 54, 3665 (2006)

[8] D. An, S. Pan, L. Huang, T. Dai, B. Krakauer, M. Zhu, ISIJ Int. 54, 422 (2014)

[9] M.R. Varma, R. Sasikumar, S.G.K. Pillai, P.K. Nair, Bull. Mater. Sci. 24, 305 (2001)

[10] D.S. Svyetlichnyy, A.I. Mikhalyov, ISIJ Int. 54, 1386 (2014)

[11] G.W. Wang, Z. Liu, J. Zhang, Adv. Mater. Res. 753-755, 932 (2013)

[12] N. Singh, doi:10.13140/RG.2.1.3625.6483.

[13] Y. Zhong, T. Zhu, ACTA Mater. 75, 337 (2014)

[14] A. Malik, G. Amberg, A. Borgenstam, J. Ågren, Model. Simul. Mater. Sci. Eng. 21, 085003 (2013)

[15] S. Saito, Y. Abe, K. Koyama, Phys. Rev. E. 96, 13317 (2017)

[16] M. Eshraghi, M. Hashemi, B. Jelinek, S. Felicelli, Metals (Basel) 7, 474 (2017)

[17] C. Xie, J. Wang, D. Wang, N. Pan, M. Wang, Commun. Comput. Phys. 17, 1037 (2015)

[18] A. Xu, W. Shyy, T. Zhao, Acta Mech. Sin. 33, 555 (2017)

[19] D. Svyetlichnyy, M. Krzyzanowski, R. Straka, L. Lach, W.M. Rainforth, Int. J. Numer. Methods Heat Fluid Flow. 28, 31 (2018)

[20] X. Zhan, Y. Wei, Z. Dong, J. Mater. Process. Technol. 208, 1 (2008)

[21] D.S. Svyetlichnyy, J. Nowak, Ł. Łach, ACRI 2012 (Lecture Notes in Computer Science, Sirakoulis G.C., Bandini S., 494 2012)

[22] W. Shen, L. Zhang, C. Zhang, Y. Xu, X. Shi, J. Mater. Eng. Perform. 25, 2065 (2016)

[23] M. Krzyzanowski, D. Svyetlichnyy, G. Stevenson, W.M. Rainforth, Int. J. Adv. Manuf. Technol. 87, 519 (2016)

[24] Ł. Łach, J. Nowak, D. Svyetlichnyy, J. Mater. Process. Technol. 255, 488 (2018)

[25] Ł. Łach, D.S. Svyetlichnyy, Mater. Res. Innov. 18, S6-295 (2014) 